\title{
Comparing the Clinical and Laboratory Features of Remitting Seronegative Symmetrical Synovitis with Pitting Edema and Seronegative Rheumatoid Arthritis
}

\author{
Misako Higashida-Konishi ${ }^{1, * \mathbb{D}}$, Keisuke Izumi ${ }^{1,2}$, Satoshi Hama ${ }^{1}$, Hiroshi Takei ${ }^{1}$, Hisaji Oshima ${ }^{1}$ \\ and Yutaka Okano ${ }^{1}$ \\ 1 Department of Connective Tissue Diseases, National Hospital Organization Tokyo Medical Center, \\ Tokyo 1528902, Japan; izz@keio.jp (K.I.); shama@ntmc-hosp.jp (S.H.); htakei@ntmc-hosp.jp (H.T.); \\ hoshimamac@mac.com (H.O.); yutakaokano@mac.com (Y.O.) \\ 2 Division of Rheumatology, Department of Internal Medicine, Keio University School of Medicine, \\ Tokyo 1608582, Japan \\ * Correspondence: higashidamisako@gmail.com; Tel.: +81-3-3411-0111
}

check for updates

Citation: Higashida-Konishi, M.; Izumi, K.; Hama, S.; Takei, H.; Oshima, H.; Okano, Y. Comparing the Clinical and Laboratory Features of Remitting Seronegative Symmetrical Synovitis with Pitting Edema and Seronegative Rheumatoid Arthritis. J. Clin. Med. 2021, 10, 1116. https:// doi.org/10.3390/jcm10051116

Academic Editor: Eugen Feist

Received: 25 January 2021

Accepted: 4 March 2021

Published: 7 March 2021

Publisher's Note: MDPI stays neutral with regard to jurisdictional claims in published maps and institutional affiliations.

Copyright: (c) 2021 by the authors. Licensee MDPI, Basel, Switzerland. This article is an open access article distributed under the terms and conditions of the Creative Commons Attribution (CC BY) license (https:/ / creativecommons.org/licenses/by/ $4.0 /)$.

\begin{abstract}
In seronegative arthritis with extremity edema, it is difficult to differentiate between remitting seronegative symmetrical synovitis with pitting edema syndrome (RS3PE) and seronegative rheumatoid arthritis (SNRA). We compared the clinical characteristics of RS3PE and SNRA in patients with and without malignancies. We retrospectively examined patients diagnosed with RS3PE (McCarty criteria) and SNRA at our hospital in 2007-2020. Malignancy was diagnosed within 2 years before or after RS3PE or SNRA diagnosis. Overall, 24 RS3PE and 124 SNRA patients were enrolled. The median ages were 79.5 and 68.5 years, and men comprised $54.2 \%$ and $37.1 \%$ of RS3PE and SNRA patients, respectively. RS3PE patients had higher inflammation levels $(p=0.004)$ and more incidences of malignancy $(p=0.034)$. Matching for age and sex, RS3PE patients had higher inflammation levels $(p=0.021)$ and more incidences of malignancy $(p=0.005)$. Overall, odds ratios (ORs) for malignancy were higher for older age (OR 1.06, $p=0.037$ ), male sex (OR 4.34, $p=0.007$ ), RS3PE patients (OR 4.83, $p=0.034$ ), and patients with extremity edema (OR 4.83, $p=0.034)$. Inflammation levels and associated factors of malignancy were higher in RS3PE patients than in SNRA patients. Patients who are older, male, with extremity edema, or had RS3PE should be screened for malignancies.
\end{abstract}

Keywords: rheumatoid arthritis; synovitis; neoplasms; edema; inflammation

\section{Introduction}

Remitting seronegative symmetrical synovitis with pitting edema (RS3PE) was first reported by McCarty et al. in 1985 [1]. It is characterized by pitting edema of the extremities, sudden onset of polyarthritis, seronegativity for rheumatoid factor (RF), excellent response to glucocorticoids, and the absence of radiologically evident erosions [1]. RS3PE mainly affects the joints of the extremities, especially the metacarpophalangeal (MCP) and proximal interphalangeal (PIP) phalanges, wrists, shoulders, elbows, knees, and ankles [2]. Although the pathophysiology of RS3PE remains unclear, vascular endothelial growth factor (VEGF) serum levels have been found to be elevated in patients with RS3PE [3]. The increase in vascular permeability by VEGF is thought to be responsible for the development of pitting edema of the dorsum of both hands and both feet in patients with RS3PE [3].

Initially, RS3PE was thought to be a type of older-onset rheumatoid arthritis (RA) [4] and was considered the same disease as seronegative RA and polymyalgia rheumatica (PMR) [5]. Subsequently, comparisons between PMR and RS3PE have been reported [6]. Kawashiri et al. reported the differences in musculoskeletal ultrasound findings of both hands between RS3PE and "seropositive" elderly onset RA; however, to our knowledge, no reports have compared the characteristics of RS3PE and "seronegative" RA [7]. 
RS3PE is often described as a paraneoplastic disease [8] and has been reported to have a high rate of malignancy development [9]. Paraneoplastic arthritis often presents as symmetrical polyarthritis, mainly affecting the wrist and fingers, and is often negative for RF and anti-cyclic citrullinated peptide antibody (ACPA) [10]. Early diagnosis of malignancy is clinically important because it improves survival. Therefore, examination for malignancy is necessary in such cases.

The primary aim of this study was to compare the clinical characteristics of RS3PE and seronegative RA and evaluate the frequency of concurrent malignancy. The secondary aim was to compare the clinical features with and without malignancies in patients with RS3PE and to compare the clinical features with and without malignancies in patients with seronegative RA.

\section{Materials and Methods}

\subsection{Compliance with Ethical Standards}

All procedures were performed in accordance with the ethical standards of the institutional and national research committees and the 1975/1983 Helsinki Declaration and its later amendments.

\subsection{Study Design}

This was a retrospective medical record study.

\subsection{Patients}

Medical records of consecutive patients diagnosed with RS3PE and seronegative RA at our hospital between 2007 and 2020 were retrospectively examined. Patients who were both ACPA- and RF-negative were included. Patients who met the criteria for both PMR and RS3PE were included in the RS3PE group and those who met the criteria for both PMR and seronegative RA were included in the seronegative RA group. PMR was diagnosed according to the 2012 European League Against Rheumatism/American College of Rheumatology (EULAR/ACR) Provisional Classification Criteria for PMR [11]. For patients diagnosed with PMR before 2012, we retrospectively reviewed whether they met the 2012 PMR classification criteria. Patients who met the criteria for both RA and RS3PE were diagnosed with RS3PE. However, those who had erosion were diagnosed with seronegative RA. We defined RS3PE and seronegative RA patients by excluding those who met the criteria for PMR as "pure RS3PE" and "pure seronegative RA." Patients who met the criteria for both RA and PMR were diagnosed with seronegative RA. Patients with paraneoplastic polyarthritis were excluded from the group of patients with RS3PE or seronegative RA. Those with distal joint swelling that rapidly disappeared after tumor resection were diagnosed with paraneoplastic polyarthritis.

\subsection{RS3PE Diagnosis}

Patients were diagnosed with RS3PE when they met the McCarty et al. criteria [1]: (1) pitting edema of the dorsum of both hands and both feet, (2) sudden onset of polyarthritis, (3) seronegative for RF, and (4) no development of radiologically evident erosions.

\subsection{Seronegative RA Diagnosis}

Seronegative RA was diagnosed according to the 2010 EULAR/ACR criteria [12]. Patients who were first diagnosed with RS3PE or PMR and later diagnosed with seronegative RA were included in the seronegative RA group.

\subsection{Clinical and Laboratory Features}

We examined the affected joints and evaluated them for systemic signs and symptoms (temperature $\geq 38.0^{\circ} \mathrm{C}$, malaise or fatigue, weight loss, morning stiffness lasting at least 1 $\mathrm{h}$, and edema). The affected joints were the shoulders, elbows, wrists, fingers (MCP and interphalangeal (IP)/PIP joints), hips, knees, ankles, and toes (MCP and IP/PIP joints). 
Edema was evaluated separately as edema of only hands, only feet, and of both limbs. We also measured the erythrocyte sedimentation rate (ESR) and the levels of C-reactive protein (CRP), hemoglobin $(\mathrm{Hb})$, albumin (Alb), lactate dehydrogenase (LDH), and matrix metalloproteinase 3 (MMP-3). Smokers were defined as those who had a smoking history within 2 years before and after RS3PE or seronegative RA diagnosis. If there were evaluable examinations, ultrasound imaging, breast imaging, joint $\mathrm{X}$-ray imaging, chest computed tomography $(\mathrm{CT})$, abdominal $\mathrm{CT}$, pelvic $\mathrm{CT}$, positron emission tomography/CT, joint magnetic resonance imaging, upper and lower gastrointestinal endoscopy, gynecological examination, and pathological tests were performed.

\subsection{Statistical Analysis}

The first analysis was performed on clinical and laboratory features of patients with RS3PE and seronegative RA. The secondary analysis was performed on the above evaluations with a 1:2 matching for age and sex. All data were analyzed using JMP version 14.0 (SAS Institute, Cary, NC, USA). The third analysis was performed to compare the clinical features of patients with or without malignancy among patients with RS3PE or seronegative RA. Univariate analysis, Fisher's exact test, and logistic regression analysis were applied to evaluate the associated factor of malignancy. A probability level less than 0.05 was used as the criterion of significance. Results that did not follow the Gaussian distribution were expressed as the median of the 25-75th percentile (interquartile range), and results that followed the Gaussian distribution were expressed as mean \pm standard deviation. The odds ratio (OR) and its 95\% confidence interval (95\% CI) indicated the increased or decreased risk of malignancy associated with a one-unit change in the predictor variable for continuous variables. For dichotomous variables, the OR indicated the risk of malignancy associated with the presence of the feature compared to the absence of the characteristic. In the case of missing data, the number of patients with available data was specified.

\section{Results}

We enrolled 24 consecutive patients with RS3PE examined at our hospital between 2007 and 2020 (Supplementary Table S1). Initially, 25 patients were diagnosed with RS3PE according to the criteria of McCarty et al. [1]. However, one patient was later diagnosed with paraneoplastic polyarthritis with rapid remission of distal swelling with pitting edema after tumor resection and was excluded from the RS3PE group. Only one patient was diagnosed with paraneoplastic polyarthritis: an 81-year-old woman who presented with polyarthritis and edema of both hands and feet. Her blood test showed high levels of CRP $(2.2 \mathrm{mg} / \mathrm{dL})$. During examination, she was diagnosed with cancer of the pancreatic body and underwent surgery to remove the body and tail of the pancreas. The postoperative course is uneventful. One month after the operation, the polyarthritis resolved and the levels of CRP decreased $(0.1 \mathrm{mg} / \mathrm{dL})$ without the use of medication.

In the control group, 124 consecutive patients with seronegative RA during the same period were enrolled. Supplementary Figure S1 shows the patient diagnosis flow.

Figure 1 shows the breakdown of patients according to the criteria for RS3PE, RA, and PMR. The RS3PE group consisted of Group A, B, and C patients. The seronegative RA group consisted of Group D and E patients. In the RS3PE and seronegative RA groups, two and 17 patients, respectively, met the 2012 EULAR/ACR provisional criteria for PMR [11] (Figure 1). After excluding those patients, 22 patients (Groups A and B, Figure 1) with RS3PE and 107 patients (Group D, Figure 1) with seronegative RA were analyzed with similar results to those obtained at baseline, including the incidence of comorbid malignancies (Supplementary Table S2). 


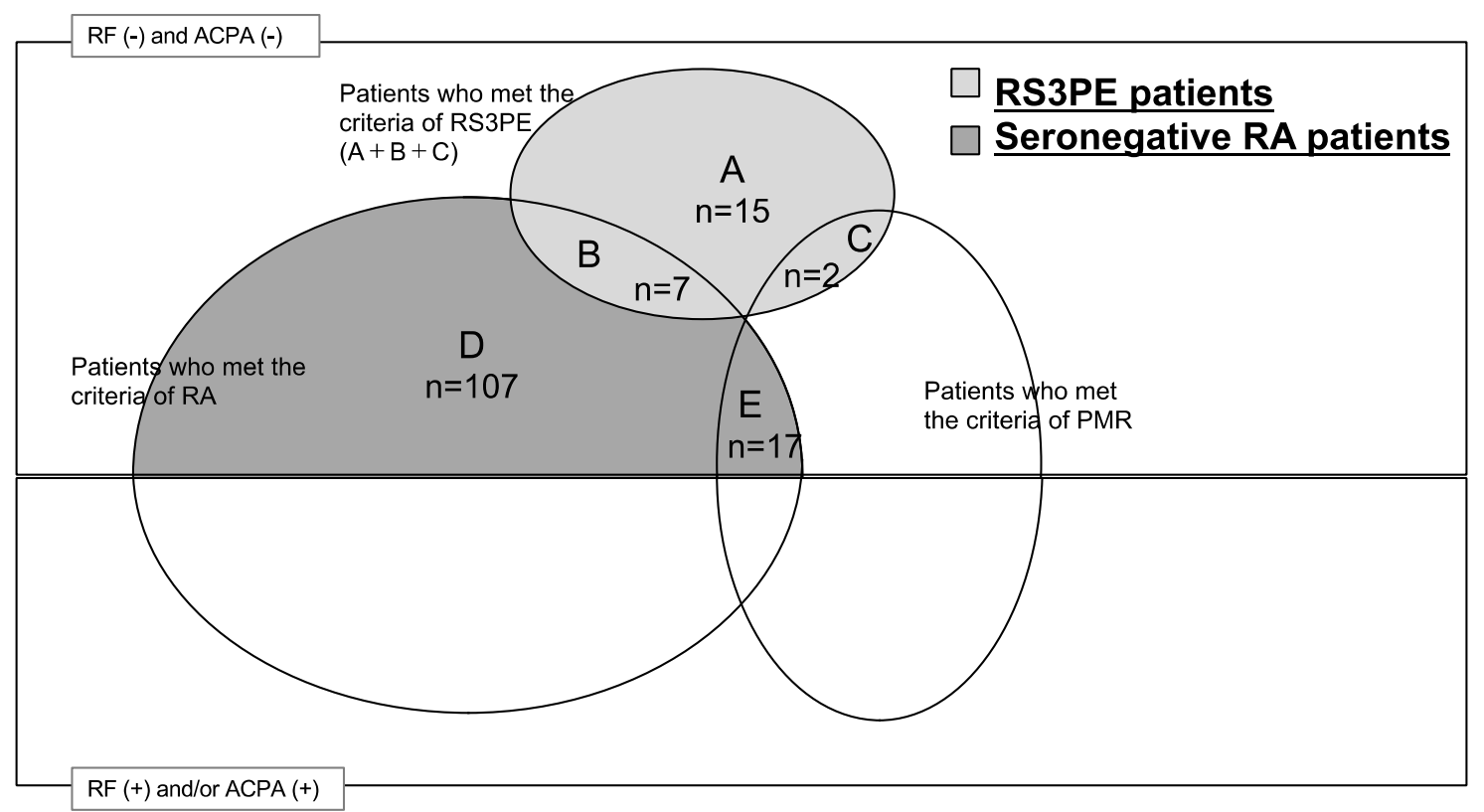

Group A: RS3PE patients who met only the criteria of RS3PE

Group B: RS3PE patients who met both the criteria of RS3PE and the criteria of RA

Group C: RS3PE patients who met both the criteria of RS3PE and the criteria of PMR

Group D: Seronegative RA patients who met only the criteria of RA

Group E: Seronegative RA patients who met both the criteria of RA and the criteria of PMR

Figure 1. Diagnostic criteria for RS3PE and seronegative RA, as used in this study. Patients in Group A met only the criteria for RS3PE. Patients in Group B met the criteria for both RS3PE and RA. Patients in Group C met the criteria for both RS3PE and PMR. Patients in Group D met only the criteria for RA. Patients in Group E met the criteria for both RA and PMR. The RS3PE group consisted of Group A + B + C patients. The seronegative RA group consisted of Group D + E patients. No patients met the criteria for RS3PE, RA, and PMR. ACPA, anti-cyclic citrullinated peptide antibody; PMR, polymyalgia rheumatica; RA, rheumatoid arthritis; RF, rheumatoid factor; RS3PE, remitting seronegative symmetrical synovitis.

\subsection{Comparison of Clinical and Laboratory Features of RS3PE and Seronegative RA}

In the first analysis, baseline characteristics at diagnosis of the 24 RS3PE patients were compared with those of the 124 seronegative RA patients (Table 1). The onset age of RS3PE was significantly higher than that of seronegative RA. The RS3PE patients had less swollen small joints and significantly higher levels of CRP, LDH, and MMP-3 than the seronegative RA patients. The numbers of swollen and/or tender joints were similar in both groups, except for the elbows and fingers, which were more affected in the seronegative RA patients. The ankles were more affected in the RS3PE patients than in the seronegative RA patients.

Malignancies were detected in six of $24(25 \%)$ patients in the RS3PE group and in eight of $124(6.5 \%)$ patients in the seronegative RA group within 2 years before and after RS3PE/seronegative RA diagnosis. The malignancy incidence rate in the RS3PE group was significantly higher than that in the seronegative RA group $(p=0.034)$. Table 2 presents the patients with malignancies and the types of malignancies. Advanced malignancies were not found in the RS3PE patients. There was one case of advanced malignancy (pancreatic cancer) in a seronegative RA patient. 
Table 1. Patient baseline characteristics at diagnosis.

\begin{tabular}{|c|c|c|c|}
\hline Characteristics & $\begin{array}{l}\text { RS3PE Patients } \\
\quad(n=24)\end{array}$ & $\begin{array}{l}\text { Seronegative RA Patients } \\
\qquad(n=124)\end{array}$ & $p$ Value \\
\hline Age, median (IQR), years & $79.5(73.8-86.5)$ & $68.5(58.5-78.0)$ & $<0.001$ \\
\hline Length of follow-up, median (IQR), months & $31.5(12.0-109.0)$ & $62.9(30.7-98.4)$ & 0.09 \\
\hline Male sex, $n(\%)$ & $13(54.2)$ & $46(37.1)$ & 0.17 \\
\hline Smoking, $n(\%)$ & $5(20.8)$ & $23(18.6)$ & 0.78 \\
\hline Diabetes, $n(\%)$ & $6(25.0)$ & $14(11.3)$ & 0.10 \\
\hline Hypertension, $n(\%)$ & $12(50.0)$ & $41(33.1)$ & 0.16 \\
\hline Hyperlipidemia, $n$ (\%) & $5(20.8)$ & $33(26.1)$ & 0.62 \\
\hline \multicolumn{4}{|l|}{ Swollen or/and tender joints, $n(\%)$} \\
\hline Shoulders & $8(33.3)$ & $67(54.3)$ & 0.08 \\
\hline Elbows & $2(8.3)$ & $53(42.7)$ & 0.001 \\
\hline Wrists & $17(70.8)$ & $100(80.7)$ & 0.28 \\
\hline Fingers & $19(79.2)$ & $120(96.8)$ & 0.022 \\
\hline Hips & $4(16.7)$ & $13(10.5)$ & 0.48 \\
\hline Knees & $9(37.5)$ & $59(47.6)$ & 0.38 \\
\hline Ankles & $18(75.0)$ & $65(52.4)$ & 0.046 \\
\hline Toes & $8(33.3)$ & $35(28.2)$ & 0.63 \\
\hline Patients with swollen large joints, $n(\%)$ & $17(70.8)$ & $64(51.6)$ & 0.12 \\
\hline Patients with swollen small joints, $n(\%)$ & $21(87.5)$ & $124(100.0)$ & 0.024 \\
\hline Number of swollen large joints, median (IQR), $n$ & $2.0(0.0-2.8)$ & $1.0(0.0-2.0)$ & 0.17 \\
\hline Number of swollen small joints, median (IQR), $n$ & $3.0(1.3-13.3)$ & $9.0(5.0-15.0)$ & 0.33 \\
\hline 28 swollen joints, median (IQR), $n$ & $4.0(1.3-10.8)$ & $8.0(5.0-14.0)$ & 0.29 \\
\hline 28 tender joints, median (IQR), $n$ & $6.5(4.3-12.0)$ & $11.0(7.3-15.0)$ & 0.15 \\
\hline Patients with erosion, $n(\%)$ & $0(0.0)$ & $39(31.5)$ & $<0.001$ \\
\hline \multicolumn{4}{|l|}{ Systemic signs and symptoms, $n(\%)$} \\
\hline Temperature $\geq 38^{\circ} \mathrm{C}$ & $2(8.3)$ & $7(5.7)$ & 0.64 \\
\hline Malaise or fatigue & $3(12.5)$ & $8(6.5)$ & 0.39 \\
\hline Weight loss & $5(20.8)$ & $12(9.7)$ & 0.16 \\
\hline $\begin{array}{l}\text { Morning stiffness } \\
\text { (lasting at least } 1 \mathrm{~h} \text { ) }\end{array}$ & $2(8.3)$ & $31(25.0)$ & 0.11 \\
\hline Edema (both hands and feet) & $24(100.0)$ & $0(0)$ & $<0.001$ \\
\hline Edema (only hands) & $0(0.0)$ & $1(0.8)$ & 1.0 \\
\hline Edema (only feet) & $0(0.0)$ & $19(15.3)$ & $<0.001$ \\
\hline CRP, median (IQR), mg/dL & $8.2(4.0-14)$ & $2.8(0.7-6.6)$ & 0.004 \\
\hline \multicolumn{4}{|l|}{ ESR, median (IQR), $\mathrm{mm} / \mathrm{h}$} \\
\hline Men+Women & $91.0(59-112.5)$ & $55.0(32.0-90.0)$ & 0.07 \\
\hline Men & $85.0(28.5-114.5)$ & $57.0(31.0-90.0)$ & 0.36 \\
\hline Women & $91.0(82-113)$ & $54.0(32.0-88.0)$ & 0.010 \\
\hline Alb, median (IQR), g/dL & $3.5(3.0-3.7)$ & $3.9(3.4-4.1)$ & 0.012 \\
\hline LDH, median (IQR), U/L & $197.0(161-234)$ & $176.0(155.5-195)$ & 0.07 \\
\hline MMP-3, median (IQR), ng/mL & & & \\
\hline
\end{tabular}


Table 1. Cont.

\begin{tabular}{|c|c|c|c|c|c|}
\hline \multirow{2}{*}{$\begin{array}{l}\text { Characteristics } \\
\text { Men+Women }\end{array}$} & \multicolumn{2}{|c|}{$\begin{array}{l}\text { RS3PE Patients } \\
\quad(n=24)\end{array}$} & \multicolumn{2}{|c|}{$\begin{array}{l}\text { Seronegative RA Patients } \\
(n=124)\end{array}$} & \multirow{2}{*}{$\begin{array}{c}\boldsymbol{p} \text { Value } \\
0.022\end{array}$} \\
\hline & $378.5(243.3-662.2)$ & $(n=16) *$ & $162.0(82.2-401.1)$ & $(n=115)^{*}$ & \\
\hline Men & $359.4(269.1-435.4)$ & $(n=7) *$ & $\begin{array}{c}211.0 \\
(115.3-420.9)\end{array}$ & $(n=45) *$ & 0.08 \\
\hline Women & 414.1(92.8-997.2) & $(n=9) *$ & $151.0(47.2-348.5)$ & $(n=70) *$ & 0.07 \\
\hline \multicolumn{6}{|l|}{$\mathrm{Hb}$, mean $\pm \mathrm{SD}, \mathrm{g} / \mathrm{dL}$} \\
\hline Men + Women & $10.7 \pm 1.8$ & & $11.9 \pm 1.8$ & & 0.024 \\
\hline Men & $10.8 \pm 2.0$ & & $12.2 \pm 1.7$ & & 0.10 \\
\hline Women & $10.6 \pm 1.5$ & & $11.7 \pm 1.8$ & & 0.12 \\
\hline $\begin{array}{l}\text { Malignancy (within } 2 \text { years before and after the } \\
\text { diagnosis of RS3PE or seronegative RA), } n(\%)\end{array}$ & $6(25.0)$ & & $8(6.5)$ & & 0.034 \\
\hline $\begin{array}{l}\text { Patients fulfilling the classification criteria for RA } \\
\qquad[11,12], n(\%)\end{array}$ & $7(29.2)$ & & $124(100.0)$ & & $<0.001$ \\
\hline $\begin{array}{l}\text { Patients fulfilling the classification criteria for } \\
\text { PMR [10], } n(\%)\end{array}$ & $2(8.3)$ & & 17 (13.7) & & 0.74 \\
\hline $\begin{array}{l}\text { Patients fulfilling the classification criteria for RA } \\
\qquad[11,12]+\text { PMR [10], } n(\%)\end{array}$ & $0(0.0)$ & & 17 (13.7) & & 0.08 \\
\hline
\end{tabular}

Alb, albumin; CRP, C-reactive protein; ESR, erythrocyte sedimentation rate; Hb, hemoglobin; IQR, inter quartile range; LDH, lactate dehydrogenase; MMP-3, matrix metalloproteinase 3; PMR, polymyalgia rheumatica; RA, rheumatoid arthritis; RS3PE, remitting seronegative symmetrical synovitis with pitting edema; SD, standard deviation. * In the case of missing data, the number of patients with available data was specified.

Table 2. Patients with malignancies 2 years before and after RS3PE or seronegative RA diagnosis.

\begin{tabular}{ccc}
\hline Sex, Age (years) & $\begin{array}{c}\text { Interval between Diagnosis of } \\
\text { RS3PE/Seronegative RA and } \\
\text { Malignancies (Months) }\end{array}$ & Malignancy Type \\
\hline RS3PE & -24 & Prostate cancer \\
\hline M, 81 & -24 & Prostate cancer \\
\hline M, 78 & -11 & Rectal cancer \\
\hline M, 78 & $0(+5$ days $)$ & Pancreatic cancer \\
\hline F, 87 & $0(+6$ days $)$ & Stomach cancer \\
\hline M, 79 & 3 & Rectal cancer \\
\hline M, 80 & & Rectal cancer \\
\hline Seronegative RA & -20 & Uterine cancer \\
\hline M, 84 & -17 & Ascending colon cancer \\
\hline F, 64 & -6 & Small cell lung cancer \\
\hline M, 82 & -5 & Breast cancer \\
\hline M, 69 & -4 & Breast cancer \\
\hline F, 58 & 1 & Diffuse large B cell lymphoma \\
\hline F, 80 & 9 & Pancreatic cancer \\
\hline M, 67 & 18 & S \\
\hline M, 83 & 9 & \\
\hline
\end{tabular}

RA, rheumatoid arthritis; RS3PE, remitting seronegative symmetrical synovitis with pitting edema; $\mathrm{M}$, male $\mathrm{F}$, female. 
3.2. Comparison of Clinical and Laboratory Features of RS3PE and Seronegative RA with a 1:2 Matching for Age and Sex

Since the incidence of malignancies depends on age and sex, we performed a 1:2 matching in the second analysis. After matching for age and sex, 24 patients with RS3PE and 48 with seronegative RA were selected for comparison. Malignancies were significantly more common in the RS3PE than in the seronegative RA patients (Table 3). The RS3PE patients had less swollen and tender joints and significantly higher CRP levels than the seronegative RA patients.

Table 3. Baseline characteristics at diagnosis of RS3PE and seronegative RA patients with a 1:2 matching for age and sex.

\begin{tabular}{|c|c|c|c|}
\hline Characteristic & $\begin{array}{l}\text { RS3PE Patients } \\
\quad(n=24)\end{array}$ & $\begin{array}{l}\text { Seronegative RA Patients } \\
\qquad(n=48)\end{array}$ & $p$ Value \\
\hline Age, median (IQR), years & $79.5(73.8-86.5)$ & $79.5(73.3-85.3)$ & 0.58 \\
\hline Male sex, $n(\%)$ & $13(54.2)$ & $23(47.9)$ & 0.80 \\
\hline \multicolumn{4}{|l|}{ Swollen or/and tender joint, $n(\%)$} \\
\hline Shoulders & $8(33.3)$ & $33(68.8)$ & 0.006 \\
\hline Elbows & $2(8.3)$ & $19(39.6)$ & 0.006 \\
\hline Wrists & $17(70.8)$ & $42(87.5)$ & 0.11 \\
\hline Fingers & $19(79.2)$ & $46(95.8)$ & 0.037 \\
\hline Hips & $4(16.7)$ & $6(12.5)$ & 0.72 \\
\hline Knees & $9(37.5)$ & $19(39.6)$ & 1.00 \\
\hline Ankles & $18(75.0)$ & $25(52.1)$ & 0.08 \\
\hline Toes & $8(33.3)$ & $11(22.9)$ & 0.40 \\
\hline Patients with swollen large joints, $n(\%)$ & $17(70.8)$ & $26(54.2)$ & 0.21 \\
\hline Patients with swollen small joints, $n(\%)$ & $21(87.5)$ & $48(100.0)$ & 0.034 \\
\hline Number of swollen small joints, median (IQR), $n$ & $3.0(1.3-13.3)$ & $9.0(6.0-15.0)$ & 0.021 \\
\hline 28 swollen joints, median (IQR), $n$ & $4.0(1.3-10.8)$ & $9.5(6.0-15.0)$ & 0.008 \\
\hline 28 tender joints, median (IQR), $n$ & $6.5(4.3-12.0)$ & $11.0(8.3-15.0)$ & 0.019 \\
\hline Patients with erosion, $n(\%)$ & $0(0.0)$ & $15(31.3)$ & 0.001 \\
\hline CRP, median (IQR), mg/dL & $8.2(4.0-14)$ & $4.4(1.3-8.4)$ & 0.021 \\
\hline ESR, median (IQR), mm/h & $91.0(59-112.5)$ & $75.0(37.0-103.0)$ & 0.25 \\
\hline LDH, median (IQR), U/L & $197.0(161-234)$ & $184.5(164.0-210.5)$ & 0.26 \\
\hline MMP-3, median (IQR), ng/mL & $378.5(243.3-662.2)$ & $251.0(124.0-555.0)$ & 0.27 \\
\hline $\mathrm{Hb}$, mean $\pm \mathrm{SD}, \mathrm{mg} / \mathrm{dL}$ & $10.7 \pm 1.8$ & $11.5 \pm 2.0$ & 0.08 \\
\hline $\begin{array}{c}\text { Malignancy } \\
\text { (within } 2 \text { years before and after the diagnosis of RS3PE or } \\
\text { seronegative RA), } n(\%)\end{array}$ & $6(25.0)$ & $1(2.1)$ & 0.005 \\
\hline Patients fulfilling the classification criteria for RA [11,12], $n(\%)$ & $7(29.2)$ & $48(100.0)$ & 0.09 \\
\hline Patients fulfilling the classification criteria for PMR [10], $n(\%)$ & $2(8.3)$ & $7(14.6)$ & 0.71 \\
\hline $\begin{array}{l}\text { Patients fulfilling the classification criteria for RA [11,12] + PMR } \\
\qquad[10], n(\%)\end{array}$ & $0(0.0)$ & $7(14.6)$ & 0.09 \\
\hline
\end{tabular}

CRP, C-reactive protein; ESR, erythrocyte sedimentation rate; Hb, hemoglobin; IQR, interquartile range; LDH, lactate dehydrogenase; MMP-3, matrix metalloproteinase 3; PMR, polymyalgia rheumatica; RA, rheumatoid arthritis; RS3PE, remitting seronegative symmetrical synovitis with pitting edema; SD, standard deviation. 


\subsection{Comparison of Clinical Features of Patients with and without Malignancies among the RS3PE} and Seronegative RA Patients

Table 4 shows a comparison of the clinical features of the patients with and without malignancies. There were 14 patients with malignancies and 134 patients without malignancies, with median ages of 79.5 and 69.5 years, respectively $(p=0.032)$. Furthermore, $71.4 \%$ and $36.6 \%$ of the patients, respectively, were men $(p=0.011)$. The RS3PE patients constituted $42.9 \%$ and $13.4 \%$ ( $p=0.034$ ) of the patients with and without malignancies, respectively. Patients with malignancies had more edema of both hands and both feet $(p=0.034)$ than those without malignancies. There was no difference between the groups in terms of percentage of patients who fulfilled the criteria for PMR $(p=1.00)$. In terms of overall ORs for malignant comorbidities among the patients with RS3PE or seronegative RA, older age (OR 1.06, 95\% CI 1.002-1.11, $p=0.037$ ), male sex (OR 4.34, 95\% CI 1.29-14.57, $p=0.007$ ), RS3PE (OR 4.83, 95\% CI 1.50-15.56, $p=0.034$ ), and edema of both hands and both feet (OR 4.83, 95\% CI 1.50-15.56, $p=0.034$ ) were associated with the presence of comorbid malignancies. Seronegative RA (OR $0.21,95 \%$ CI 0.06-0.07, $p=0.034$ ) and increased $\mathrm{Hb}$ levels in men (OR $0.51,95 \%$ CI $0.33-0.81, p=0.005$ ) were associated with the absence of comorbid malignancies (Table 5).

Table 4. Patient baseline characteristics at diagnosis of RS3PE and seronegative RA patients with or without malignancies.

\begin{tabular}{|c|c|c|c|}
\hline Characteristics & $\begin{array}{l}\text { With Malignancy } \\
\qquad(n=14)\end{array}$ & $\begin{array}{l}\text { Without Malignancy } \\
\qquad(n=134)\end{array}$ & $p$ Value \\
\hline Age, median (IQR), years & $79.5(68.5-82.3)$ & $69.5(60.0-79.0)$ & 0.032 \\
\hline Length of follow-up, median (IQR), months & $40.6(7.9-87.7)$ & $57.4(27.4-97.7)$ & 0.36 \\
\hline Male sex, $n(\%)$ & $10(71.4)$ & $49(36.6)$ & 0.011 \\
\hline Smoking, $n(\%)$ & $5(35.7)$ & $23(17.2)$ & 0.14 \\
\hline Diabetes, $n(\%)$ & $4(28.6)$ & $16(11.9)$ & 0.10 \\
\hline Hypertension, $n(\%)$ & $5(35.7)$ & $48(35.8)$ & 1.00 \\
\hline Hyperlipidemia, $n(\%)$ & $4(28.6)$ & $34(25.4)$ & 0.76 \\
\hline \multicolumn{4}{|l|}{ Swollen or/and tender joints, $n(\%)$} \\
\hline Shoulders & $5(35.7)$ & $70(52.2)$ & 0.27 \\
\hline Elbows & $5(35.7)$ & $50(37.3)$ & 1.00 \\
\hline Wrists & $11(78.6)$ & $106(79.1)$ & 1.00 \\
\hline Fingers & $13(92.9)$ & $126(94.0)$ & 1.00 \\
\hline Hips & $2(14.3)$ & $15(11.2)$ & 0.67 \\
\hline Knees & $5(35.7)$ & $63(47.0)$ & 0.56 \\
\hline Ankles & $8(57.1)$ & $75(56.0)$ & 1.00 \\
\hline Toes & $4(28.6)$ & $39(29.1)$ & 1.00 \\
\hline Patients with swollen large joints, $n(\%)$ & $6(42.9)$ & $75(56.0)$ & 0.41 \\
\hline Patients with swollen small joints, $n(\%)$ & $13(92.9)$ & $132(98.0)$ & 0.26 \\
\hline Number of swollen large joints, median (IQR), $n$ & $0.0(0.0-2.3)$ & $1.0(0.0-2.0)$ & 0.44 \\
\hline Number of swollen small joints, median (IQR), $n$ & $12.5(4.3-18.5)$ & $8.0(4.0-13.0)$ & 0.46 \\
\hline 28 swollen joints, median (IQR), $n$ & $9.5(3.5-16.8)$ & $8.0(4.0-12.0)$ & 0.62 \\
\hline 28 tender joints, median (IQR), $n$ & $7.5(5.8-19.3)$ & $10.0(7.0-14.3)$ & 0.74 \\
\hline Patients with erosion, $n(\%)$ & $5(35.7)$ & $34(25.4)$ & 0.52 \\
\hline
\end{tabular}


Table 4. Cont.

\begin{tabular}{|c|c|c|c|}
\hline \multirow[b]{2}{*}{ Systemic signs and symptoms, $n(\%)$} & $\begin{array}{l}\text { With Malignancy } \\
\quad(n=14)\end{array}$ & $\begin{array}{l}\text { Without Malignancy } \\
\qquad(n=134)\end{array}$ & \multirow[t]{2}{*}{$p$ Value } \\
\hline & & & \\
\hline Temperature $\geq 38^{\circ} \mathrm{C}$ & $0(0.0)$ & $9(6.7)$ & 1.00 \\
\hline Malaise or fatigue & $2(14.3)$ & $9(6.7)$ & 0.28 \\
\hline Weight loss & $1(7.1)$ & $16(12.0)$ & 1.00 \\
\hline Morning stiffness (lasting at least $1 \mathrm{~h}$ ) & $4(28.6)$ & $29(21.7)$ & 0.55 \\
\hline Edema (both hands and feet) & $6(42.9)$ & $18(13.4)$ & 0.034 \\
\hline Edema (only hands) & $0(0.0)$ & $1(0.8)$ & 1.00 \\
\hline Edema (only feet) & $0(0.0)$ & $19(14.2)$ & 0.22 \\
\hline CRP, median (IQR), mg/dL & $6.1(3.1-11.9)$ & $3.1(0.8-7.2)$ & 0.08 \\
\hline \multicolumn{4}{|l|}{ ESR, median (IQR), mm/h } \\
\hline Men + Women & $46.0(21.5-112.0)$ & $59.0(33.0-91.5)$ & 0.88 \\
\hline Men & $90.0(35.0-114.0)$ & $59.0(31.0-90.5)$ & 0.53 \\
\hline Women & $22.5(13.8-91.3)$ & $59.0(33.5-93.5)$ & 0.15 \\
\hline Alb, median (IQR), g/dL & $3.5(3.1-4.0)$ & $3.8(3.3-4.1)$ & 0.24 \\
\hline LDH, median (IQR), U/L & $174.5(166.8-214.8)$ & $\begin{array}{c}178.0 \\
(155.0-206.3)\end{array}$ & 0.79 \\
\hline \multicolumn{4}{|l|}{ MMP-3, median (IQR), ng/mL } \\
\hline Men+Women & $220.0(43.8-364.8)$ & $181.0(84.8-428.5) \quad(n=118) *$ & 0.75 \\
\hline Men & $234.7(133.0-364.8)$ & $\begin{array}{c}213.0 \\
(116.0-426.2) \\
\end{array}$ & 0.85 \\
\hline Women & $37.7(28.1-463.8)$ & $162.0(66.8-465.0)$ & 0.13 \\
\hline \multicolumn{4}{|l|}{$\mathrm{Hb}$, mean $\pm \mathrm{SD}, \mathrm{g} / \mathrm{dL}$} \\
\hline Men + Women & $10.9 \pm 2.0$ & $11.8 \pm 1.8$ & 0.10 \\
\hline Men & $10.3 \pm 1.4$ & $12.3 \pm 1.7$ & 0.001 \\
\hline Women & $12.7 \pm 2.3$ & $11.5 \pm 1.8$ & 0.24 \\
\hline Patients diagnosed with RS3PE, $n(\%)$ & $6(42.9)$ & $18(13.4)$ & 0.034 \\
\hline Patients diagnosed with RA $[11,12], n(\%)$ & $8(57.1)$ & $116(86.6)$ & 0.034 \\
\hline $\begin{array}{l}\text { Patients fulfilling the classification criteria for RA } \\
\qquad[11,12], n(\%)\end{array}$ & $10(71.4)$ & $121(90.0)$ & 0.058 \\
\hline $\begin{array}{l}\text { Patients fulfilling the classification criteria for } \\
\qquad \text { PMR }[10], n(\%)\end{array}$ & $1(7.1)$ & $18(13.4)$ & 1.00 \\
\hline
\end{tabular}

Alb, albumin; CRP, C-reactive protein; ESR, erythrocyte sedimentation rate; $\mathrm{Hb}$, hemoglobin; IQR, interquartile range; $\mathrm{LDH}$, lactate dehydrogenase; MMP-3, matrix metalloproteinase 3; RA, rheumatoid arthritis; RS3PE, remitting seronegative symmetrical synovitis with pitting edema; SD, standard deviation. * In the case of missing data, the number of patients with available data was specified. 
Table 5. Risk factors for malignancy in patients with RS3PE or seronegative RA analyzed by univariate logistic regression analysis.

\begin{tabular}{|c|c|c|c|}
\hline Characteristics & Odds Ratio & 95\% Confidence Interval & $p$ Value \\
\hline Age & 1.06 & $1.002-1.11$ & 0.037 \\
\hline Length of follow-up & 0.999801 & $0.9994-1.0002$ & 0.36 \\
\hline Male sex & 4.34 & $1.29-14.57$ & 0.007 \\
\hline Smoking & 2.68 & $0.82-8.74$ & 0.10 \\
\hline Diabetes & 2.95 & $0.83-10.52$ & 0.10 \\
\hline Hypertension & 0.995 & $0.32-3.14$ & 0.99 \\
\hline Hyperlipidemia & 1.18 & $0.35-3.997$ & 0.79 \\
\hline \multicolumn{4}{|l|}{ Swollen or/and tender joints } \\
\hline Shoulders & 0.51 & $0.16-1.60$ & 0.25 \\
\hline Elbows & 0.93 & $0.30-2.94$ & 0.91 \\
\hline Wrists & 0.97 & $0.25-3.71$ & 0.96 \\
\hline Fingers & 0.83 & $0.10-7.13$ & 0.86 \\
\hline Hips & 1.32 & $0.27-6.49$ & 0.73 \\
\hline Knees & 0.63 & $0.20-1.97$ & 0.42 \\
\hline Ankles & 1.05 & $0.34-3.19$ & 0.93 \\
\hline Toes & 0.97 & $0.29-3.29$ & 0.97 \\
\hline Patients with swollen large joints & 0.59 & $0.19-1.79$ & 0.35 \\
\hline Patients with swollen small joints & 0.20 & $0.02-2.32$ & 0.20 \\
\hline Number of swollen large joints & 0.87 & $0.60-1.25$ & 0.44 \\
\hline Number of swollen small joints & 1.04 & $0.98-1.10$ & 0.22 \\
\hline 28 swollen joints & 1.03 & $0.95-1.11$ & 0.50 \\
\hline 28 tender joints & 1.004 & $0.92-1.10$ & 0.92 \\
\hline Patients with erosion & 1.63 & $0.51-5.21$ & 0.41 \\
\hline \multicolumn{4}{|l|}{ Systemic signs and symptoms } \\
\hline Temperature $\geq 38^{\circ} \mathrm{C}$ & $8.20 \times 10^{-7}$ & $0->10^{6}$ & 0.99 \\
\hline Malaise or fatigue & 2.31 & $0.45-11.97$ & 0.32 \\
\hline Weight loss & 0.57 & $0.07-4.63$ & 0.60 \\
\hline $\begin{array}{l}\text { Morning stiffness } \\
\text { (lasting at least } 1 \mathrm{~h} \text { ) }\end{array}$ & 1.45 & $0.42-4.96$ & 0.56 \\
\hline Edema (both hands and feet) & 4.83 & $1.50-15.56$ & 0.034 \\
\hline Edema (only hands) & $6.45 \times 10^{-7}$ & $0->10^{5}$ & 0.99 \\
\hline Edema (only feet) & $2.78 \times 10^{-7}$ & $0->10^{6}$ & 0.99 \\
\hline CRP & 1.08 & $1.18-0.92$ & 0.08 \\
\hline \multicolumn{4}{|l|}{ ESR } \\
\hline Men+Women & 0.999905 & $0.98-1.02$ & 0.08 \\
\hline Men & 1.006 & $0.988-1.02$ & 0.51 \\
\hline Women & 0.98 & $0.95-1.01$ & 0.25 \\
\hline $\mathrm{Alb}$ & 0.63 & $0.24-1.65$ & 0.35 \\
\hline $\mathrm{LDH}$ & 1.0009 & $0.99-1.02$ & 0.90 \\
\hline
\end{tabular}


Table 5. Cont.

\begin{tabular}{cccc}
\hline Characteristics & Odds Ratio & 95\% Confidence Interval & $p$ Value \\
\hline MMP-3 & & & \\
\hline Men +Women & 1.00009 & $0.9992-1.001$ & 0.84 \\
\hline Men & 1.0006 & $0.9993-1.002$ & 0.34 \\
\hline Women & 0.9985 & $0.99-1.003$ & 0.50 \\
\hline Hb & 0.77 & & 0.11 \\
\hline Male + Women & 0.51 & $0.57-1.06$ & 0.005 \\
\hline Men & 1.47 & $0.80-2.71$ & 0.21 \\
\hline Women & 4.83 & $1.50-15.56$ & 0.034 \\
\hline Patients with RS3PE & 0.21 & $0.06-0.07$ & 0.034 \\
\hline $\begin{array}{c}\text { Patients with seronegative RA } \\
\text { Patients fulfilling the classification } \\
\text { criteria for RA [11,12] }\end{array}$ & 0.27 & $0.07-0.98$ & 0.046 \\
\hline $\begin{array}{c}\text { Patients fulfilling the classification } \\
\text { criteria for PMR [10] }\end{array}$ & 0.50 & $0.06-4.02$ & 0.51 \\
\hline $\begin{array}{c}\text { Patients fulfilling the classification } \\
\text { criteria for RA [11,12] + PMR [10] }\end{array}$ & $2.82 \times 10^{-7}$ & $0->10^{6}$ & 0.99 \\
\hline
\end{tabular}

Alb, albumin; CRP, C-reactive protein; ESR, erythrocyte sedimentation rate; $\mathrm{Hb}$, hemoglobin; IQR, interquartile range, $\mathrm{LDH}$, lactate dehydrogenase; MMP-3, matrix metalloproteinase 3; PMR, polymyalgia rheumatica; RA, rheumatoid arthritis; RS3PE, remitting seronegative symmetrical synovitis with pitting edema; SD, standard deviation.

\subsection{Comparison of Baseline Characteristics between RS3PE Patients with and} without Malignancies

No clinical differences were noted between the RS3PE patients with and without malignancies (Supplementary Table S3).

\subsection{Comparison of Baseline Characteristics between Seronegative RA Patients with and without Malignancies}

The seronegative RA patients with malignancies had less swollen large joints $(p=0.027)$, lower MMP-3 levels (83.8 vs. $173.0 \mathrm{ng} / \mathrm{mL}, p=0.07)$, lower ESRs in women (19.0 vs. $55.0 \mathrm{~mm} / \mathrm{h}, p=0.020)$, and higher $\mathrm{Hb}$ levels in women $(13.7 \pm 1.3$ vs. $11.6 \pm 1.8, p=0.045)$ than those without malignancies (Supplementary Table S4).

\section{Discussion}

\subsection{Comparison of Clinical and Laboratory Features of RS3PE and Seronegative RA}

We found that patients with RS3PE were characterized by an older age at onset, higher affectation of the ankles compared to the elbows and fingers, higher levels of CRP and ESR, and a higher malignancy rate compared to patients with seronegative RA. These results (Table 1) are similar to those of Olive et al. [2], who reported that, in RS3PE patients, the MCP (81.5\%) and PIP joints (70.4\%), wrists (55.5\%), shoulders $(48 \%)$, knees $(33.3 \%)$, and ankles (25.9\%) were more frequently affected, while the elbows $(11.1 \%)$ were less frequently affected. Patients with RS3PE had swollen and/or tender finger joints less frequently than those with seronegative RA $(79.2 \%$ vs. $96.8 \%, p=0.022)$. The reason for this is that patients with seronegative RA must present with 11 or more swollen or tender joints, including at least one small joint, to meet the 2010 EULAR/ACR criteria for RA [12]. This suggests that patients with seronegative RA tend to have many small joints affected. In our study, RS3PE more frequently affected the joints of the ankles than did seronegative RA. The high incidence of affected joints of the ankles in RS3PE patients may be due to attending physicians determining swelling in the ankle because of lower extremity edema in RS3PE patients. 
The number of affected joints in the RS3PE patients was lower than that in the seronegative RA patients; however, the levels of CRP, and MMP-3 were higher. When analyzed with a 1:2 matching for age and sex, CRP levels were higher in the RS3PE group than in the seronegative RA group, while MMP-3 levels were comparable between the groups (Table 3). This implies that RS3PE and seronegative RA are essentially different diseases. Patients with RS3PE have often been reported to be positive for human leukocyte antigen (HLA)-B7, -Cw7, and -DQw2 [13], but not for HLA-DRB1, which is positive in RA [13,14]. Furthermore, RS3PE patients have higher levels of VEGF than RA patients [3]. This suggests that the pathogenesis of RS3PE is different from that of seronegative RA. Malignancies such as advanced cancers [15] and kidney cancers [16], which cause high levels of CRP, were not found in the RS3PE patients in this study.

PMR and seronegative RA have both positive HLA-DRB1, which may suggest that their etiologies may be the same; however, there are differences regarding their clinical manifestations. In PMR patients, there is significantly more frequent bilateral shoulder and hip pain and significantly less frequent peripheral arthritis (peripheral synovitis) than in RA patients [11]. Based on the distribution of the affected joints, it is not difficult to distinguish PMR from seronegative RA. Therefore, when the primary symptom of a patient who meets the criteria for PMR is peripheral arthritis; a diagnosis of RA is often made when the patient also meets the criteria for RA.

Compared to RS3PE, PMR has also been found to be significantly more common in male patients with a higher frequency of hip morning stiffness and pain [6]. Salvarani et al. [17] reported 19 cases of PMR with distal extremity swelling with pitting edema. However, edema in both hands and both feet was present in only three of the 19 cases, and all three cases met the criteria for RS3PE [1], although there are some missing data on RF. PMR with distal extremity swelling with pitting edema appears to identify a more benign disease subset than PMR without edema [18]. Patients who met the criteria for both PMR and RS3PE have previously been categorized as RS3PE [6,19]. Therefore, PMR with edema in all extremities could have been defined as RS3PE.

In our study, the patients who met the criteria for both RS3PE and PMR were defined as having RS3PE, and those who met the criteria for both seronegative RA and PMR were defined as having seronegative RA. Two (8.3\%) and 17 (13.7\%) patients with RS3PE and seronegative RA met the criteria for PMR [11], respectively. Excluding these patients who met the criteria for PMR, we reanalyzed 22 "pure RS3PE" and 107 "pure seronegative RA" patients. There were no differences in clinical characteristics and results between the "pure RS3PE" and "pure seronegative RA" groups, including the incidence of comorbid malignancies. These results suggest that it is not possible to differentiate RS3PE from seronegative RA regardless of the patients meeting the criteria for PMR. In paraneoplastic syndromes in rheumatology, musculoskeletal symptoms are known to occur in the joints and muscles [20] and PMR-like symptoms are also known to develop [21]. In our study, however, there was no relationship between meeting the PMR criteria and the presence or absence of malignancies (Table 5).

\subsection{Comparison between RS3PE/Seronegative RA with and without Malignancies}

Comorbid malignancies were found in $25.0 \%$ and $6.5 \%$ of the RS3PE and seronegative RA patients, respectively (Table 1). Based on data from the National Cancer Institute of Japan [22], the 4-year incidences of malignancies (2 years before and after the diagnosis of RS3PE/seronegative RA) in the Japanese population of the same age were $9.1 \%$ and $6.3 \%$ in RS3PE and seronegative RA patients, respectively. Thus, compared with the Japanese population, the incidence of comorbid malignancies was higher in the RS3PE group and comparable in the seronegative RA group. This is consistent with the findings of a previous report that the incidence of malignancies is higher in patients with RS3PE than in the general population [9]. The types of malignancies associated with RS3PE [23] include stomach, rectal, and prostate cancers, as observed in our study. 


\subsection{Comparison between RS3PE Patients with and without Malignancies}

In the current study, there was no significant difference in the clinical characteristics of RS3PE between patients with and without malignancies (Supplementary Table S3). Origuchi et al. reported that RS3PE with malignancies has higher MMP-3 serum levels than RS3PE without malignancies, due to the abundant production of MMP-3 owing to malignancies [24]. In our study, there was no difference in MMP-3 levels. This discrepancy may have been due to the small number of cases both in the study by Origuchi et al. [24] and ours. These authors included eight patients with malignancy out of a total of 33 patients with RS3PE, and our study included six patients with malignancy out of a total of 24 patients with RS3PE. Due to the small number of cases to be analyzed, sufficient detection power may not have been obtained. These authors also included not only patients with edema of the hands and feet, but also that of only hands or only feet, which is different from our inclusion criteria that included patients with edema in both hands and both feet, similar to the study of McCarty et al. [1]. There was no difference in MMP-3 levels when analyzed separately by sex.

\subsection{Comparison between Seronegative RA Patients with and without Malignancies}

In our study, the seronegative RA patients with malignancies had lower MMP-3 levels and fewer swollen large joints than those without malignancies. Although MMP-3 serum levels can be elevated with steroids [25], all patients in this study had not used steroids before seronegative RA diagnosis. Additionally, patients with malignancies had fewer swollen large joints than those without malignancies (Supplementary Table S4). Serum levels of MMP-3 have been reported to be higher in RA patients with synovitis in large joints [26]. The MMP-3 serum levels did not correlate with the number of tender and swollen joints used in the core set of ACR, but they correlated with the Lansbury's joint scores, which have a high coefficient for large joints [27]. Therefore, in our study, the low circulating levels of MMP-3 in seronegative RA patients with malignancy may be due to the small number of swollen large joints.

\subsection{Comparison between Seronegative RA and RS3PE Patients with and without Malignancies}

We also examined the differences in the clinical characteristics of the overall patients with and without malignant comorbidities. The ORs of the patients with malignancies were higher for older age, male sex, RS3PE, and edema of both hands and both feet (Table 5). Regarding older and male patients, these results are consistent with data from the National Cancer Institute of Japan and the general Japanese trend. The high ORs of RS3PE and edema in both hands and both feet for malignancy also suggest that a thorough examination for malignancies should be performed in patients with RS3PE.

\subsection{Limitations}

Our study has several limitations. First, this was a retrospective study. Therefore, we employed matching to minimize selection bias. Second, 23 seronegative RA patients (one with malignancy, 22 without malignancies) and eight RS3PE patients (three with malignancies, five without malignancies) could not be followed for $\geq 2$ years after the diagnosis of seronegative RA and RS3PE, respectively. Nevertheless, the results were not different after the exclusion of these patients. In our study, the incidence of malignancies was defined within 2 years before and after RS3PE or seronegative RA diagnosis; however, it is not clear within what year malignancy should be included. Some reports included comorbid malignancies within a definite period after the onset of RS3PE [6,24], while other reports did not present a definite period $[9,28]$. The significant difference in the incidence of comorbid malignancies between the RS3PE and seronegative RA groups was noted even when including malignancies within 1 or 3 years before or after the diagnosis of RS3PE/RA. Third, our study population was small. Since RS3PE is a rare disease and this was a single center study, multicenter validation studies are warranted. Finally, there 
were some missing data on Alb and MMP-3, but there were no missing data on important indices such as CRP and ESR.

\section{Conclusions}

Patients with RS3PE had higher CRP levels and a higher risk for malignancy than those with seronegative RA. As RS3PE patients are likely to have malignancies, it is necessary to thoroughly examine for malignancies at RS3PE diagnosis.

The seronegative RA patients with malignancies had lower MMP-3 levels and fewer swollen large joints at RA diagnosis than those without malignancy. Furthermore, among seronegative RA patients, it is recommended that patients with lower MMP-3 levels and fewer swollen large joints should be screened for malignancy.

These findings may enable the performance of a differential diagnosis between RS3PE and seronegative RA. Moreover, this may encourage clinicians to examine for malignancies in patients with RS3PE, contributing to improved patient outcomes.

Supplementary Materials: The following are available online at https:/ /www.mdpi.com/2077-038 3/10/5/1116/s1, Figure S1: Flow of patient diagnosis, Table S1: Clinical features of the 24 patients with RS3PE at the time of diagnosis, Table S2: Baseline characteristics at diagnosis of RS3PE and seronegative RA patients, excluding patients fulfilling the classification criteria for PMR, Table S3. Baseline characteristics in patients with RS3PE at diagnosis, Table S4: Baseline characteristics in patients with seronegative RA at diagnosis.

Author Contributions: Conceptualization, M.H.-K. and K.I.; methodology, M.H.-K.; software, M.H.K.; validation, M.H.-K., K.I., S.H., H.T., and H.O.; formal analysis, M.H.-K.; investigation, M.H.-K.; data curation, M.H.-K.; writing_original draft preparation, M.H.-K.; writing—review and editing, M.H.-K.; visualization, M.H.-K.; supervision, Y.O.; project administration, M.H.-K. All authors have read and agreed to the published version of the manuscript.

Funding: This research received no external funding.

Institutional Review Board Statement: The study was conducted according to the guidelines of the Declaration of Helsinki, and approved by the Ethics Committee of National Hospital Organization Tokyo Medical Center (approval number R19-011 and date of approval: 2 March 2015).

Informed Consent Statement: Informed consent was waived due to the retrospective study de-sign.

Data Availability Statement: Not available.

Acknowledgments: The authors thank Manami Koyama for her assistance in data collection.

Conflicts of Interest: The authors declare no conflict of interest.

\section{References}

1. McCarty, D.J.; O’Duffy, J.D.; Pearson, L.; Hunter, J.B. Remitting seronegative symmetrical synovitis with pitting edema. RS3PE syndrome. JAMA 1985, 254, 2763-2767. [CrossRef]

2. Olivé, A.; Del Blanco, J.; Pons, M.; Vaquero, M.; Tena, X. The clinical spectrum of remitting seronegative symmetrical synovitis with pitting edema. The Catalán Group for the Study of RS3PE. J. Rheumatol. 1997, 24, 333-336.

3. Arima, K.; Origuchi, T.; Tamai, M.; Iwanaga, N.; Izumi, Y.; Huang, M.; Tanaka, F.; Kamachi, M.; Aratake, K.; Nakamura, H.; et al. RS3PE syndrome presenting as vascular endothelial growth factor associated disorder. Ann. Rheum. Dis. 2005, 64, $1653-1655$. [CrossRef]

4. Mavragani, C.P.; Moutsopoulos, H.M. Rheumatoid arthritis in the elderly. Exp. Gerontol. 1999, 34, 463-471. [CrossRef]

5. Healy, L.A. RS3PE syndrome. J. Rheumatol. 1990, 17, 414.

6. Kimura, M.; Tokuda, Y.; Oshiawa, H.; Yoshida, K.; Utsunomiya, M.; Kobayashi, T.; Deshpande, G.A.; Matsui, K.; Kishimoto, M. Clinical characteristics of patients with remitting seronegative symmetrical synovitis with pitting edema compared to patients with pure polymyalgia rheumatica. J. Rheumatol. 2012, 39, 148-153. [CrossRef] [PubMed]

7. Kawashiri, S.; Suzuki, T.; Okada, A.; Tsuji, A.; Takatani, A.; Shimizu, T.; Koga, T.; Iwamoto, N.; Ichinose, K.; Nakamura, H.; et al. Differences in musculoskeletal ultrasound findings between RS3PE syndrome and elderly-onset rheumatoid arthritis. Clin. Rheumatol. 2020, 39, 1981-1988. [CrossRef] [PubMed]

8. Sibilia, J.; Friess, S.; Schaeverbeke, T.; Maloisel, F.; Bertin, P.; Goichot, B.; Kuntz, J.L. Remitting seronegative symmetrical synovitis with pitting edema (RS3PE): A form of paraneoplastic polyarthritis? J. Rheumatol. 1991, 26, 115-120. 
9. Elizabeth, B.R. Remitting seronegative symmetrical synovitis with pitting edema syndrome: Follow up for neoplasia. J. Rheumatol. 2005, 32, 1760-1761.

10. Morel, J.; Deschamps, V.; Toussirot, E.; Pertuiset, E.; Sordet, C.; Kieffer, P.; Berthelot, J.M.; Champagne, H.; Mariette, X.; Combe, B. Characteristics and survival of 26 patients with paraneoplastic arthritis. Ann. Rheum. Dis. 2008, 67, 244-247. [CrossRef]

11. Dasgupta, B.; Cimmino, M.A.; Maradit-Kremers, H.; Schmidt, W.A.; Schirmer, M.; Salvarani, C.; Bachta, A.; Dejaco, C.; Duftner, C.; Jensen, H.S.; et al. Provisional classification criteria for polymyalgia rheumatica: A European League Against Rheumatism/American College of Rheumatology collaborative initiative. Ann. Rheum. Dis. 2012, 71, 484-492. [CrossRef]

12. Aletaha, D.; Neogi, T.; Silman, A.J.; Funovits, J.; Felson, D.T.; Bingham, C.O., 3rd; Birnbaum, N.S.; Burmester, G.R.; Bykerk, V.P.; Cohen, M.D.; et al. Rheumatoid arthritis classification criteria: An American College of Rheumatology/European League Against Rheumatism collaborative initiative. Arthritis Rheum. 2010, 62, 2569-2581. [CrossRef]

13. Yao, Q.; Su, X.; Altman, R.D. Is remitting seronegative symmetrical synovitis with pitting edema (RS3PE) a subset of rheumatoid arthritis? Semin. Arthritis Rheum. 2010, 40, 89-94. [CrossRef] [PubMed]

14. Gregersen, P.K.; Silver, J.; Winchester, R.J. The shared epitope hypothesis. An approach to understanding the molecular genetics of susceptibility to rheumatoid arthritis. Arthritis Rheum. 1987, 30, 1205-1213. [CrossRef] [PubMed]

15. Fan, Z.; Fan, K.; Gong, Y.; Huang, Q.; Yang, C.; Cheng, H.; Jin, K.; Ni, Q.; Yu, X.; Luo, G.; et al. The CRP/Albumin Ratio Predicts Survival and Monitors Chemotherapeutic Effectiveness in Patients With Advanced Pancreatic Cancer. Cancer Manag. Res. 2019, 11, 8781-8788. [CrossRef]

16. Saito, K.; Kihara, K. Role of C-reactive protein as a biomarker for renal cell carcinoma. Expert Rev. Anticancer Ther. 2010, 10, 1979-1989. [CrossRef]

17. Salvarani, C.; Gabriel, S.; Hunder, G. Distal extremity swelling with pitting edema in polymyalgia rheumatica. Report of nineteen cases. Arthritis Rheum. 1996, 39, 73-80. [CrossRef]

18. Salvarani, C.; Cantini, F.; Macchioni, P.; Olivieri, I.; Niccoli, L.; Padula, A.; Boiardi, L. Distal musculoskeletal manifestations in polymyalgia rheumatica: A prospective followup study. Arthritis Rheum. 1998, 41, 1221-1226. [CrossRef]

19. Bucaloiu, I.D.; Olenginski, T.P.; Harrington, T.M. Remitting seronegative symmetrical synovitis with pitting edema syndrome in a rural tertiary care practice: A retrospective analysis. Mayo Clin. Proc. 2007, 82, 1510-1515. [CrossRef]

20. Manger, B.; Schett, G. Paraneoplastic syndromes in rheumatology. Nat. Rev. Rheumatol. 2014, 10, 662-670. [CrossRef]

21. Muller, S.; Hider, S.; Helliwell, T.; Partington, R.; Mallen, C. The real evidence for polymyalgia rheumatica as a paraneoplastic syndrome. Reumatismo 2018, 70, 23-34. [CrossRef]

22. Cancer Statistics. Available online: https://ganjoho.jp/reg_stat/statistics/stat/index.html (accessed on 9 September 2020). (In Japanese).

23. Emamifar, A.; Hess, S.; Gildberg-Mortensen, R.; Hansen, I.M.J. Association of remitting seronegative symmetrical synovitis with pitting edema, polymyalgia rheumatica, and adenocarcinoma of the prostate. Am. J. Case Rep. 2016, 17, 60-64. [CrossRef] [PubMed]

24. Origuchi, T.; Arima, K.; Kawashiri, S.Y.; Tamai, M.; Yamasaki, S.; Nakamura, H.; Tsukada, T.; Aramaki, T.; Furuyama, M.; Miyashita, T.; et al. High serum matrix metalloproteinase 3 is characteristic of patients with paraneoplastic remitting seronegative symmetrical synovitis with pitting edema syndrome. Mod. Rheumatol. 2012, 22, 584-588. [CrossRef] [PubMed]

25. Sharif, M.; Salisbury, C.; Taylor, D.J.; Kirwan, J.R. Changes in biochemical markers of joint tissue metabolism in a randomized controlled trial of glucocorticoid in early rheumatoid arthritis. Arthritis Rheum. 1998, 41, 1203-1209. [CrossRef]

26. Ohuchi, E.; Iwata, K.; Yamanaka, H. Serum MMP-3 in rheumatoid arthritis. Inflamm. Regenerat. 2004, 24, 154-160. (In Japanese) [CrossRef]

27. Nagasawa, H.; Kameda, H.; Amano, K.; Takeuchi, T. Clinical significance of serum matrix metalloproteinase-3 level in patients with rheumatoid arthritis. Inflamm. Regenerat. 2004, 25, 60-64. (In Japanese) [CrossRef]

28. Karmacharya, P.; Donato, A.A.; Aryal, M.R.; Ghimire, S.; Pathak, R.; Shah, K.; Shrestha, P.; Poudel, D.; Wasser, T.; Subedi, A.; et al. RS3PE revisited: A systematic review and meta-analysis of 331 cases. Clin. Exp. Rheumatol. 2016, 34, 404-415. 